Real Analysis Exchange

Vol. 24(2), 1998/9, pp. 599-605

Krzysztof Ciesielski* Department of Mathematics, West Virginia University, Morgantown, WV 26506-6310, USA e-mail: KCies@wvnvms. wvnet.edu

Kenneth R. Kellum, Department of Mathematics and Computer Science, San José State University, San José, California 95192-0103 e-mail:

kellum@sjsumcs.sjsu.edu

\title{
COMPOSITIONS OF DARBOUX AND CONNECTIVITY FUNCTIONS
}

\begin{abstract}
We describe here an example of a Darboux function $k$ from the unit interval $\mathcal{I}=[0,1]$ onto itself such that $k$ is not the composition of any finite collection of connectivity functions from $\mathcal{I}$ into $\mathcal{I}$. This answers a question of Ceder [2].
\end{abstract}

In [6] the second author proved that there exists a connectivity function from $\mathcal{I}$ into $\mathcal{I}$ which cannot be written as the composition of finitely many almost continuous functions. In the present paper we show that the techniques developed in the earlier paper can be extended to answer a question of Ceder [2]. We prove that there exits a Darboux function which cannot be factored into a finite composition of connectivity functions. This stands in contrast with the following fact.

Proposition 1. (Natkaniec [8]) Assume that $\mathcal{I}$ is not a union of less than continuum many of its meager subsets. Then every function from I into I with dense level sets can be expressed as the composition of two almost continuous functions.

Since every almost continuous function $f: \mathcal{I} \rightarrow \mathcal{I}$ is connectivity, this theorem shows that our example must be relatively nice. (Cannot have dense level sets.)

\footnotetext{
Key Words: connectivity function, Darboux function, composition.

Mathematical Reviews subject classification: Primary 26A15, 26A30; Secondary 54C30, $54 \mathrm{C} 08$

Received by the editors April 27, 1998

* The first author was partially supported by NSF Cooperative Research Grant INT9600548 with its Polish part financed by KBN.

Papers authored or co-authored by a Contributing Editor are managed by a Managing Editor or one of the other Contributing Editors.
} 
Our terminology is standard and follows [3]. In particular, functions will be identified with their graphs. Recall also the following definitions. (See [5] for more on these.) A function $f: X \rightarrow Y$ from a topological space $X$ into a topological space $Y$ is Darboux if $f[C]$ is connected in $Y$ whenever $C \subset X$ is connected in $X ; f$ is a connectivity function if the restriction $f \nmid C$ of $f$ to $C$ is a connected subset of $X \times Y$ for every connected subset $C$ of $X$. We will be interested in these functions only when $X=Y=\mathcal{I}$. Then $f: \mathcal{I} \rightarrow \mathcal{I}$ is Darboux if an $f$-image of any interval is an interval. Clearly every connectivity function $f: \mathcal{I} \rightarrow \mathcal{I}$ is Darboux.

For $U \subset \mathbb{R}$ we will write $\operatorname{int}(U)$ to denote the interior of $U$.

Recall also that connectivity can be characterized in terms of continua, where a continuum is a compact connected set. We say that a continuum $M \subset X \times Y$ cuts the function $f: X \rightarrow Y$ if $M \cap f=\emptyset$ and there exist points $\langle x-1, y-1\rangle$ and $\left\langle x_{2}, y_{2}\right\rangle$ in $M$ such that $f\left(x_{1}\right)<y_{1}$ and $f\left(x_{2}\right)>y_{2}$. A function $f: \mathcal{I} \rightarrow \mathcal{I}$ is a connectivity function if and only if no continuum in $\mathcal{I}^{2}$ cuts $f$. (See e.g. [4].)

\section{The Example}

Let $C$ denote the Cantor middle two-fifths set.

$$
C=\left\{\sum_{n=1}^{\infty} \frac{i_{n}}{5^{n}}: i_{n} \in\{0,2,4\} \text { for every } n\right\} .
$$

Geometrically, $C$ is obtained from $\mathcal{I}$ by first removing the pair of intervals $(1 / 5,2 / 5)$ and $(3 / 5,4 / 5)$ from $\mathcal{I}$, then by removing similar pairs of intervals (the middle two fifths) from each of $[0,1 / 5],[2 / 5,3 / 5]$, and $[4 / 5,1]$, etc. Let $\mathbb{P}_{L}$ denote the set of closures of removed intervals which are the left members of a removed pair and $\mathbb{P}_{R}$ the set of closures of right members of removed pairs. (For example $[1 / 5,2 / 5] \in \mathbb{P}_{L}$ and $[3 / 5,4 / 5] \in \mathbb{P}_{R}$.) Thus $\mathbb{P}=\mathbb{P}_{L} \cup \mathbb{P}_{R}$ is the family of all closures of components of $\mathcal{I} \backslash C$. Also, let $\Delta$ denote the diagonal $\{\langle x, x\rangle: x \in \mathcal{I}\}$ in $\mathcal{I} \times \mathcal{I}$ and let $C^{\circ}$ denote the points of $C$ which are not endpoints of the removed intervals; that is, $C^{\circ}=\mathcal{I} \backslash \cup \mathbb{P}$. We define a function $k: \mathcal{I} \rightarrow \mathcal{I}$ in the following way.

On $C^{\circ}$ we define $k$ so that $k\left[C^{\circ}\right]=C^{\circ}, k\left\lceil C^{\circ}\right.$ is one-to-one, and $x \neq k(x)$ for each $x \in C^{\circ}$. Thus, $\Delta \cap k\left\lceil C^{\circ}=\emptyset\right.$.

Next, suppose that $P$ and $Q$ are the closures of adjacent removed middle fifths and suppose that $P \in \mathbb{P}_{L}$. We define $k$ on $P \cup Q$ such that $k[P \cup Q]=\mathcal{I}$, $k \uparrow P$ and $k \uparrow Q$ are each continuous and strictly increasing and so that $k \uparrow P$ lies above $\Delta$ while $k\lceil Q$ lies below $\Delta$. So, $\Delta \cap k=\emptyset$. 


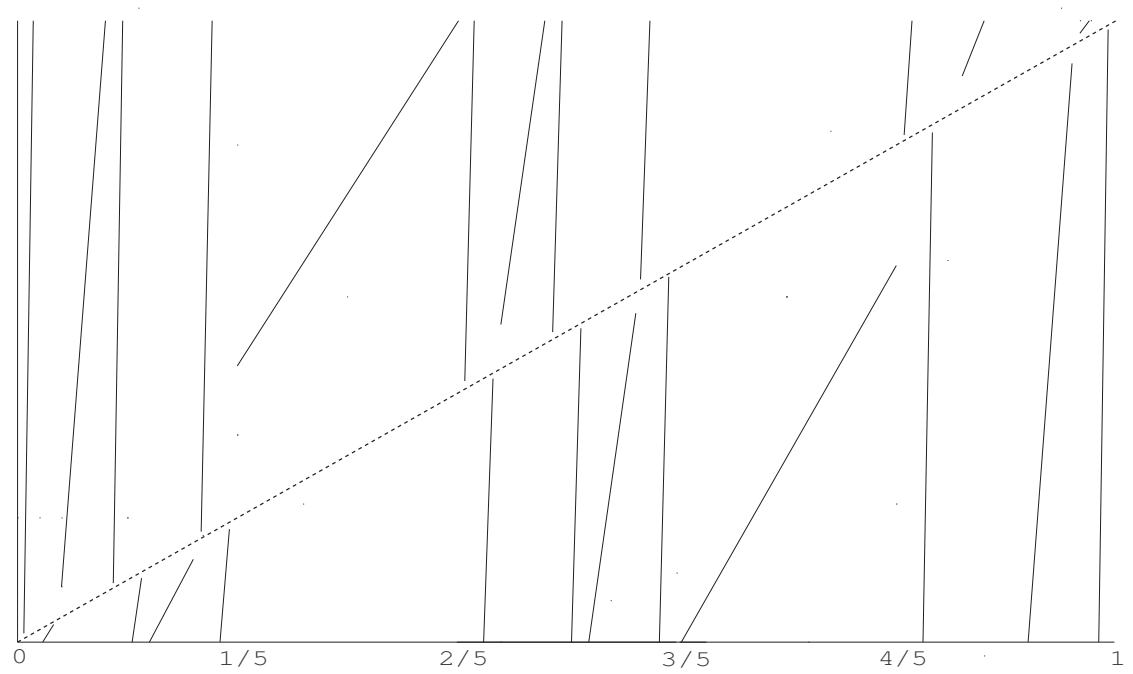

Figure 1: The function $k$ (the dotted line represents $\Delta$ ).

Clearly, $k$ is not a connectivity function since its graph is separated by $\Delta$. It is also easy to see that $k$ is Darboux. Indeed, if an interval $J \subset \mathcal{I}$ is a subset of a closure $P$ of some interval from $\mathbb{P}$, then $k[J]$ is also an interval since $k \uparrow P$ is continuous. But otherwise $J$ contains a pair $P$ and $Q$ of closures of adjacent removed middle fifths from the construction of $C$ and so $k[J]=\mathcal{I}$ is also an interval.

Now we are ready for the main result of the paper.

Theorem 1. If $k=g \circ f$, where $f$ and $g$ are functions from $\mathcal{I}$ onto itself, $f$ is connectivity, and $g$ is Darboux, then $f$ is a homeomorphism.

Proof. First note that

if $U \in \mathbb{P}$, then $f\lceil U$ and $g\lceil f[U]$ are homeomorphisms.

Indeed, since $k \uparrow U$ is one-to-one so then is $f \uparrow U$. Thus $f \uparrow U$ is a Darboux function with closed level sets and so (see e.g. [1, thm 5.2]) it is continuous. So, $f \uparrow U$ is a homeomorphism. By the same reasoning $g \uparrow f[U]$ also is a homeomorphism.

Next note that

$f[C]$ does not contain any non-trivial interval. 
Indeed, suppose for a moment that $f[C]$ contains a non-trivial interval, say $V$. Since $g[V]$ is connected, this would imply that an uncountable subset of $C$ is mapped by $k$ onto a connected set, a contradiction.

Notice also that

$$
\text { if } U \in \mathbb{P}_{L} \text { and } V \in \mathbb{P}_{R} \text {, then } f[\operatorname{int}(U)] \cap f[\operatorname{int}(V)]=\emptyset .
$$

To see it assume, by way of contradiction, that there are $U=\left[u_{1}, u_{2}\right] \in \mathbb{P}_{L}$ and $V=\left[v_{1}, v_{2}\right] \in \mathbb{P}_{R}$ such that $f[\operatorname{int}(U)]$ and $f[\operatorname{int}(V)]$ do overlap. Then, by (1), $g\lceil(f[U] \cup f[V])$ is a homeomorphism, say increasing. Thus $f\lceil U$ and $f \uparrow V$ are increasing as well. Moreover, from the choice of $U$ and $V, g$ maps $f[U] \cup f[V]$ onto $\mathcal{I}, g \circ f\left(u_{2}\right)=k\left(u_{2}\right)=1$, and $g \circ f\left(v_{1}\right)=k\left(v_{1}\right)=0$. So $h=\left(g\lceil f[U] \cup f[V])^{-1}\right.$ is an increasing homeomorphism on $\mathcal{I}$. Note also that $f(x) \neq h(x)$ for every $x \in \mathcal{I}$, since otherwise $k(x)=g(f(x))=g(h(x))=x$ contradicting the fact that $k \cap \Delta=\emptyset$. Therefore $h \cap f=\emptyset$. Moreover, $f\left(v_{1}\right)=h(0)<h\left(v_{1}\right)$ and $f\left(u_{2}\right)=h(1)>h\left(u_{2}\right)$ since $h$ is increasing. Thus, the graph of $h$, which is a continuum, cuts $f$, contradicting the connectivity of $f$. Condition (3) has been proved.

For $P \in \mathbb{P}$ let $c_{P} \in P$ be the end-point of $P$ for which $k\left(c_{P}\right) \in\{0,1\}$ and let $e_{P}=f\left(c_{P}\right)$. So, by (1), (3), and the construction of $k$ we see that for every $P \in \mathbb{P}$ and $\mathcal{P} \subseteq\{U \in \mathbb{P}: f[\operatorname{int}(U)] \cap f[\operatorname{int}(P)] \neq \emptyset\}$

$$
e_{P} \in f[U] \text { for every } U \in \mathcal{P} \text {, }
$$

$$
f[\bigcup \mathcal{P}] \text { is an interval with one end point equal to } e_{P},
$$

and

$$
g \text { is a homeomorphism on } f[\bigcup \mathcal{P}] \text {. }
$$

In what follows, we say that a family $\mathcal{P} \subset \mathbb{P}$ is dense in an open interval $(a, b) \subset \mathcal{I}$ if $\bigcup \mathcal{P} \subset(a, b), a=\inf \bigcup \mathcal{P}, b=\sup \bigcup \mathcal{P}$, and between any two members of $\mathcal{P}$ there is another. The main technical fact in the proof is the following.

$(\star)$ Assume that $f[\operatorname{int}(P)] \cap f[\operatorname{int}(Q)] \neq \emptyset$ for some different $P, Q \in \mathbb{P}$ and let $\mathcal{P}$ be the set of all $U \in \mathbb{P}$ between $P$ and $Q$ with $f[\operatorname{int}(U)] \cap f[\operatorname{int}(P)] \neq \emptyset$. Then the family $\mathcal{P}$ is non-empty and dense in $(a, b)$, where $a=\inf \bigcup \mathcal{P}$ and $b=\sup \bigcup \mathcal{P}$. Moreover, there exists a homeomorphism $h$ into $K=$ $f[\bigcup \mathcal{P}]$ such that either

(i) $e_{P} \in K \subset\left[e_{P}, 1\right]$ and the graph of $f\lceil(a, b)$ is below $h$, or

(ii) $e_{P} \in K \subset\left[0, e_{P}\right]$ and the graph of $f \uparrow(a, b)$ is above $h$. 
and either

(iii) the domain of $h$ is $[0, b)$ or

(iv) the domain of $h$ is $(a, 1]$

Indeed, to see that $\mathcal{P}$ is non-empty and dense in $(a, b)$ assume, to the contrary, that there are $U$ and $V$ in $\mathcal{P} \cup\{P, Q\}$ with no member of $\mathcal{P}$ between them. Suppose $U$ is to the left of $V$ and let $[c, d]=f[U \cap V]$. By (2) we can choose $y \in(c, d) \backslash f\left[C^{\circ}\right]$. Also, by (1), we can also choose $p \in U$ and $q \in V$ such that the continuum $[p, q] \times\{y\}$ is disjoint with $f \uparrow U$ and $f \uparrow V$. Thus $[p, q] \times\{y\}$ cuts the function $f$ since, by (6), either both $f \uparrow U$ and $f \uparrow V$ are increasing or both are decreasing. But this contradicts the connectivity of $f$.

To see the existence of $h$ as in $(\star)$ assume that $P \in \mathbb{P}_{R}$ and that $f \uparrow P$ is increasing, the other three cases being similar. We will show that this implies (i) and (iii). Under this assumption we have $k[\bigcup \mathcal{P}]=[0, b)$, since there are elements of $\mathcal{P}$ arbitrarily close to $b$. Also $K=\left[e_{P}, d\right)$ for some $d>e_{P}$ and, by $(6), g \uparrow K$ is an increasing homeomorphism between $K$ and $[0, b)$. Let $h=\left(g\lceil K)^{-1}\right.$ and notice that $f(x) \neq h(x)$ for every $x \in[a, b]$, since otherwise $k(x)=g(f(x))=g(h(x))=x$ contradicting the fact that $k \cap \Delta=\emptyset$. Thus $h \cap(f \uparrow(a, b))=\emptyset$ and, by the connectivity of $f \uparrow(a, b)$, its graph lies either below or above $h$. It is below, since $e_{P} \in f[\bigcup \mathcal{P}]$ is the minimal value of $h$. This proves $(\star)$.

Now, if $f[\operatorname{int}(P)] \cap f[\operatorname{int}(Q)]=\emptyset$ for any different $P, Q \in \mathbb{P}$, then $f$ is one-to-one on $\bigcup\{\operatorname{int}(P): P \in \mathbb{P}\}$. It is also one-to-one on $C^{\circ}$, since so is $k$. Therefore $f^{-1}(z)$ has at most two points for every $z$ and so it is closed. Thus, $f$ is a continuous (see e.g. [1, thm 5.2]) and so, it must be a homeomorphism. So, by way of contradiction, assume that there are different $P, Q \in \mathbb{P}$ for which $f[\operatorname{int}(P)] \cap f[\operatorname{int}(Q)] \neq \emptyset$.

It follows from (3) that either $P, Q \in \mathbb{P}_{L}$ or $P, Q \in \mathbb{P}_{R}$. We will assume that $P, Q \in \mathbb{P}_{L}$ and that $f$ is increasing on $P$, the other cases being similar. Let $a<b, \mathcal{P}, K$, and $h$ be as in $(\star)$. Then case (i) holds. Also, by (3), there exists $P^{\prime} \in \mathbb{P}_{R}, P^{\prime} \subset(a, b)$, such that $f\left[P^{\prime}\right] \cap K=\emptyset$. Notice that $f\left[P^{\prime}\right]$ is below $K$, as $f \uparrow(a, b)$ is below $h$, and that

there is $Q^{\prime} \in \mathbb{P} \backslash\left\{P^{\prime}\right\}, Q^{\prime} \subset(a, b)$, such that $f\left[\operatorname{int}\left(P^{\prime}\right)\right] \cap f\left[\operatorname{int}\left(Q^{\prime}\right)\right] \neq \emptyset$.

To see (7) assume, to the contrary, that such a $Q^{\prime}$ does not exist. By (2) we can choose $y \in f\left[P^{\prime}\right] \backslash f[C]$. Pick $c \in P^{\prime}$ with $\langle c, y\rangle$ above $f \uparrow P^{\prime}$ and note that if $f \uparrow P^{\prime}$ is increasing, then $[a, c] \times\{y\}$ cuts $f$, and if $f \uparrow P^{\prime}$ is decreasing, then $[c, b] \times\{y\}$ cuts $f$. (Recall that $\mathcal{P}$ is dense in $(a, b)$ and $K$ is above $f\left[P^{\prime}\right]$.) This contradiction proves (7). 
Next, assume that $P^{\prime}$ is below $Q^{\prime}$ let $a^{\prime}<b^{\prime}, \mathcal{P}^{\prime}, K^{\prime}$, and $h^{\prime}$ satisfy $(\star)$. Notice that $\left(a^{\prime}, b^{\prime}\right) \subset(a, b)$ and that $f \uparrow\left(a^{\prime}, b^{\prime}\right)$ must be above $h^{\prime}$. Only two possibilities remain.

CAse 1. There is $P^{\prime \prime} \in \mathbb{P}$ such that $P^{\prime \prime} \subset\left(a^{\prime}, b^{\prime}\right)$ and $f\left[P^{\prime \prime}\right] \cap\left(K \cup K^{\prime}\right)=\emptyset$.

Then, as in (7), we can find another $Q^{\prime \prime} \in \mathbb{P} \backslash\left\{P^{\prime \prime}\right\}$ such that $Q^{\prime \prime} \subset\left(a^{\prime}, b^{\prime}\right)$ and $f\left[P^{\prime \prime}\right] \cap f\left[Q^{\prime \prime}\right] \neq \emptyset$. Using $(\star)$ again, we can find appropriate $a^{\prime \prime}<b^{\prime \prime}, \mathcal{P}^{\prime \prime}$, $K^{\prime \prime}$, and $h^{\prime \prime}$. The range of $h^{\prime \prime}$ is strictly between $K$ and $K^{\prime}$, and, since the domain of $h^{\prime \prime}$ contains either 0 or $1, h^{\prime \prime}$ cuts $f$, a contradiction.

CASe 2. $f\left[P^{\prime \prime}\right] \subset K \cup K^{\prime}$ for every $P^{\prime \prime} \in \mathbb{P}$ with $P^{\prime \prime} \subset\left(a^{\prime}, b^{\prime}\right)$.

Note that $K \cap K^{\prime}=\emptyset$. So $J=\left(e_{P^{\prime}}, e_{P}\right)$ is non-empty. Take $y \in J \backslash f[C]$, which exists by (2). Then $\left[a^{\prime}, b^{\prime}\right] \times\{y\}$ cuts $f$, a contradiction.

This last contradiction completes the proof.

Corollary 1. The function $k$ cannot be written as a finite composition of connectivity functions from $\mathcal{I}$ onto itself.

Proof. Assume $k$ is such a composition and let $n$ be the smallest integer such that $k=f_{n} \circ \cdots \circ f_{1}$, where each of $f_{1} \ldots f_{n}$ is a connectivity function from $\mathcal{I}$ onto $\mathcal{I}$. Since $f_{n} \circ \cdots \circ f_{2}$ is Darboux, by Theorem $1, f_{1}$ is continuous. By Theorem 4 of [7], $f_{2} \circ f_{1}$ is connectivity, contradicting the definition of $n$.

\section{References}

[1] A. M. Bruckner and J. Ceder, Darboux Continuity, Jahresber. Deutsch. Math.-Verein. 67 (1965), 93-117.

[2] J. G. Ceder, On composition with connected functions, Real Analysis Exch. 11 (1985-86), 380-390.

[3] K. Ciesielski, Set Theory for the Working Mathematician, London Math. Soc. Student Texts 39, Cambridge Univ. Press 1997.

[4] B. D. Garrett, D. Nelms, and K. R. Kellum, Characterizations of connected real functions, Jber. Deutsch. Math.-Verein. 73 (1971), 131-137.

[5] R. G. Gibson and T. Natkaniec, Darboux like functions, Real Analysis Exch. 22(2) (1996-97), 492-533. (Preprint ${ }^{\star}$ available. $^{1}$ )

[6] K. R. Kellum, Compositions of Darboux-like functions, Real Analysis Exch., 23 (1997-98), 211-216.

\footnotetext{
${ }^{1}$ Preprints marked by * are available in electronic form from: Set Theoretic Analysis Web Page: http://www.math.wvu.edu/homepages/kcies/STA/STA.html
} 
[7] K. R. Kellum and H. Rosen Compositions of continuous functions and connected functions, Proc. Amer. Math. Soc. 115 (1992), 145-149.

[8] T. Natkaniec, On compositions and products of almost continuous functions, Fund. Math. 139 (1991), 59-74. 